\title{
The junior doctor view of undergraduate ophthalmology education
}

\author{
Shahzaib Rehan ${ }^{1}$
}

Received: 1 February 2018 / Accepted: 7 February 2018 / Published online: 23 February 2018

(c) The Royal College of Ophthalmologists 2018

I read medicine at Cardiff University and graduated with honours in 2016. The curriculum was lecture based and ophthalmology was afforded 1 week during year 4 of the programme. Since then, a new case-based learning strategy has been deployed for the overall curriculum, with ophthalmology retaining 1 week of the dedicated teaching time. Now, as a practising junior doctor, I am in a position to reflect back and assess whether my undergraduate ophthalmology training (UOT) adequately equipped me for clinical practice.

The risk of common eye pathologies such as glaucoma, cataract and age-related macular degeneration (AMD) increases with age. The population is ageing and this will see a rise in the incidence and prevalence of eye disease. It is imperative that UOT is taught well, as today's students will be providing tomorrow's eye care. Ophthalmology skills are core clinical skills and are not only relevant to specialist trainees but also to ward-based juniors and primary care providers. Despite this, globally, dedicated UOT time within curricula is reducing, as a consequence of several factors including time, teachers and resources $[1,2]$.

My 1 week of the dedicated UOT at medical school consisted largely of outpatient clinic visits with ad hoc teaching, two theatre sessions and a limited number of lectures. Clinics included glaucoma, paediatric ophthalmology, cataract, AMD and eye casualty. The emphasis appeared to be 'on the job training', being delivered to two medical students at a time, by the practitioners in clinic. Some of the environments created by the supervising doctors were engaging, stimulating and encouraged active participation via history taking and practising clinical skills; including core ones such as assessing pupillary reactions and fundoscopy using a direct ophthalmoscope. However,

Shahzaib Rehan

drshazrehan@gmail.com

1 Ophthalmology Department, University Hospital of Wales, Heath Park Way, Health Park, Cardiff CF14 4XW, UK such environments and the feeling of doctors 'caring about your development' were not always encountered. These variable experiences, secondary to the lack of standardisation of practitioners, ultimately impacted on the acquisition of my theoretical knowledge and understanding and my practical skills.

At the end of my UOT week, I felt stressed by the amount of work that I was expected to have covered, had little in the way of lecture notes, and felt disappointed. Being actively taught by experts within a field is a privilege and I believe it can spark enthusiasm within students. That being said, the skill of self-directed learning and study is imperative at university. Despite appreciating this, I do believe that programme directors have a responsibility to ensure that students get taught the basics well. At times during medical school, I felt that I was paying costly admission fees 'to teach myself'. Once basic concepts and understanding have been attained, arguably further facilitation of learning is largely what is required of clinical teachers and not 'spoon feeding'. My UOT week was inadequate and left me with a large list of curriculum items to cover and a poor understanding of basic ophthalmological principles and concepts. Going into the junior doctor foundation programme (2 years of clinical training postgraduation in the UK), had it not been for student-selected components and my elective in Ophthalmology, I would not have felt prepared to encounter eye problems on the ward. My additional experiences enabled me to develop a systematic ward-based approach to examining eyes with a good appreciation of when and how urgently to refer to ophthalmology.

Good ophthalmology training is a must and should be delivered to all medical students. This will help to improve the understanding and confidence in assessing and managing eye conditions and inspire the ophthalmologists of tomorrow. A combination approach to teaching ophthalmology (lectures, case-based discussion, clinic time, elearning and simulation), to cater to the different learning styles that are preferred by students, should be deployed in addition to, I feel, a minimum of 2 weeks of UOT. 
Moreover, certain skills that are encountered within the ophthalmology block, i.e., direct fundoscopy, should be made into core compulsory clinic skills and should be practiced (as per auscultation of the heart and lungs) over the entirety of one's medical course. It baffles me that a medical school and foundation programme requirement as a core skill is 'preparing and administering IV medications', which in clinical practice is a task that is carried out on a daily basis by nursing colleagues. Whereas, as doctors, we are expected to carry out direct ophthalmoscopy to screen for potentially sight and life-threatening conditions, yet one can be signed off as a competent foundation programme doctor potentially being incompetent at performing this skill. Simulation and virtual reality (VR) using web-based methods can augment and enhance learning. Furthermore, setting up such systems can benefit programme directors because they do not need to recruit patients for the interactive learning of cases, it eases time constraints, and can it expose students to a wide breadth of conditions and depth of knowledge [1]. The way postgraduate clinical medicine is being taught is changing, and various technologies, as described above are being incorporated into the curricula. It is time for undergraduate medicine to do the same.

\section{Compliance with ethical standards}

Conflict of interest The author declares that he has no conflict of interest.

\section{References}

1. Succar T, Grigg J, Beaver HA, Lee AG. A systematic review of best practices in teaching ophthalmology to medical students. Surv Ophthalmol. 2016;61:83-94.

2. Jacobs DS. Teaching doctors about the eye: trends in the education of medical students and primary care residents. Surv Ophthalmol. 1998;42:383e9. 